\title{
Early changes of left ventricular filling pattern after reperfused ST-elevation myocardial infarction and doxycycline therapy: Insights from the TIPTOP trialis
}

\author{
Giampaolo Cerisano ${ }^{\mathrm{a}, *}$, Piergiovanni Buonamici ${ }^{\mathrm{a}}$, Guido Parodi ${ }^{\mathrm{a}}$, Alberto Santini ${ }^{\mathrm{a}}$, Guia Moschi $^{\mathrm{a}}$, \\ Renato Valenti ${ }^{\text {, }}$, Angela Migliorini ${ }^{\mathrm{a}}$, Paolo Colonna ${ }^{\mathrm{b}}$, Benedetta Bellandi ${ }^{\mathrm{a}}$, \\ Anna Maria Gori ${ }^{c}$, David Antoniucci ${ }^{\text {a }}$ \\ a Cardiovascular and Thoracic Department, Careggi Hospital, Florence, Italy \\ ${ }^{\mathrm{b}}$ Division of Cardiology, Hospital Policlinico of Bari, Bari, Italy \\ c Department of Experimental and Clinical Medicine, University of Florence, Italy
}

\section{A R T I C L E I N F O}

Article history:

Received 18 October 2016

Accepted 27 March 2017

Available online $\mathrm{xxxx}$

\section{Keywords:}

Acute myocardial infarction

Diastolic function

Doxycycline therapy

Left ventricular remodeling

Primary percutaneous coronary intervention

\begin{abstract}
A B S T R A C T
Aim: Metalloproteinases inhibition by doxycycline reduces cardiac protein degradation at extracellular and intracellular level in the experimental model ischemia/reperfusion injury. Since both extracellular cardiac matrix and titin filaments inside the cardiomyocyte are responsible for the myocardial stiffness, we hypothesized that doxycycline could favorably act on left ventricular (LV) filling pressures in patients after reperfused acute ST-elevation myocardial infarction (STEMI).

Methods and results: Seventy-three of 110 patients of the TIPTOP trial underwent a 2D-Echo-Doppler on admission, and at pre-discharge and at 6-month after a primary PCI for STEMI and LV dysfunction. From admission to pre-discharge, LV filling changed from a high filling pressure (HFP) to a normal filling pressure (NFP) pattern in $91 \%$ of the doxycycline-group, and in $67 \%$ of the control-group. Conversely, $1 \%$ of the doxycycline-group, and $37 \%$ of the control-group changed the LV filling from NFP to HFP pattern. Overall, a pre-discharge HFP pattern was present in 4 patients (11\%) of the doxycycline-group and in 13 patients (36\%) of the control-group ( $p=$ $0.025)$. The evaluation of metalloproteinases and their tissue inhibitors plasma concentrations provide possible favorable action of doxycycline. On the multivariate analyses, troponine I peak $(p=0.026)$, doxycycline $(p=$ $0.033)$, and on admission to pre-discharge LVEF changes ( $p=0.044$ ) were found to be associated with predischarge HFP pattern. Independently of their baseline LV filling behavior, the 6-month remodeling was less in patients with pre-discharge NFP pattern than in patients with HFP pattern.

Conclusions: In patients with STEMI and LV dysfunction doxycycline can favorably modulate the LV filling pattern early after primary $\mathrm{PCI}$.
\end{abstract}

(c) 2017 Elsevier B.V. All rights reserved.
Doppler echocardiography has provided a rapid, feasible, and simple noninvasive method of assessing left ventricular (LV) filling in various cardiac diseases in which diastolic abnormalities have been observed [1]. In this context, a growing body of clinical evidence have indicated

Abbreviations: STEMI, ST-elevation myocardial infarction; MMPs, mealloproteinases; NFP, normal filling pressure; HFP, high filling pressure; $E$-wave, transmitral early peak velocity; A-wave, transmitral late peak velocity; DT, deceleration time of transmitral early peak velocity; E', early peak velocity on the septal side of the mitral annulus; Vp, left ventricular flow propagation velocity during early filling; LVEDVi, left ventricular end diastolic volume index; LVESVi, left ventricular end systolic volume index; LVEF, left ventricular ejection fraction; ECM, extracellular cardiac matrix; PCI, percutaneous coronary intervention.

it The study was not supported by any public grant or industry support, none of the authors has financial disclosures.

* Corresponding author at: Largo Brambilla 3, I-50141 Florence, Italy.

E-mail address: giampaolo.cerisano@gmail.com (G. Cerisano). that the presence of echocardiographic indexes of elevated LV filling pressures, as a marker of LV diastolic dysfunction, predicts a poor prognosis early after acute myocardial infarction (AMI) [2], despite pharmacological [3] or mechanical reperfusion [4,5] and independently of systolic function [2]. Thus, a major unresolved question is how to optimally manage AMI patients having advanced LV diastolic dysfunction [6].

For the first time in the clinical setting, the TIPTOP (Early Short-term Doxycycline Therapy In Patients with Acute Myocardial Infarction and Left Ventricular Dysfunction to Prevent The Ominous Progression to Adverse Remodeling) trial [7] demonstrated that a timely, short-term therapy with doxycycline is safe and able to induce a significant decrease in LV dilation in patients successfully treated with primary percutaneous coronary intervention ( $\mathrm{pPCI}$ ) for a first ST-elevation AMI (STEMI) and LV dysfunction. Several pleiotropic properties of 
doxycycline [8] mainly related to its ability to act as a matrix metalloproteinases (MMPs) inhibitor at the level of extracellular collagen matrix (ECM) [9] could explain this favorable anti-remodeling effect observed in the experimental $[10,11]$ and clinical [12] setting. Metalloproteinases activation can also occur intracellularly in heart subjected to ischemia/reperfusion (I/R), and it is responsible for the degradation of sarcomeric proteins including titin [13-15], thus contributing to cardiomyocytes injury under oxidative stress. Accordingly, MMPs inhibition by doxycycline prevents acute stunning in isolated rat-hearts subjected to I/R injury $[13,14]$ and mimics the MMP-2 inhibition and infarct size reduction found with ischemic post-conditioning [16]. Since both ECM and titin filaments inside the cardiomyocyte are two important components responsible for the myocardial stiffness, it is possible to hypothesize that doxycycline, through its MMPs-inhibitor action, has a favorable effect on diastolic LV filling.

Thus, we set out to analyze the time course of changes in the LV filling pattern during the acute stage of STEMI treated with doxycycline in the TIPTOP trial.

\section{Methods}

\subsection{Study design, patients and procedures}

The TIPTOP study design has been already descripted in detail [7]. In brief, TIPTOP trial is a prospective, phase-2, single-centre, randomized, open-label controlled trial in which 110 patients older than 18 years with acute STEMI and LV ejection fraction (LVEF) $<40 \%$ were randomly assigned in a 1:1 ratio to receive doxycycline or standard care. All patients were treated with primary $\mathrm{PCI}$, including stenting of the infarct-related artery (IRA), and received medical therapy for STEMI and LV dysfunction in accordance with standard and recommended practice. Doxycycline (Bassado; Pfizer Italia s.r.l.) was administered at $100 \mathrm{mg}$ oral dose immediately after primary PCI and then every $12 \mathrm{~h}$ for 7 days. The antimicrobial dose we used ensures plasma levels of doxycycline similar to those obtained with higher doses used in the experimental model where the doxycycline has been proved effective in inhibiting MMPs and preventing abdominal aortic aneurysm [17] and postinfarction LV remodeling [10,11]. The pre-defined primary endpoint of the TIPTOP trial was the percent change from baseline to 6 months in echocardiographic LV enddiastolic volume index (LVEDVi). Thus, two-dimensional and Doppler echocardiographic examinations (2D-Echo-Doppler) at baseline (immediately after primary $\mathrm{PCI}$ ) and at 6 months were performed. DICOM standardized images were recorded and stored on digital media, and sent to an independent, blinded, off-site core laboratory for analysis. The study plan also included: i) ${ }^{99 \mathrm{~m}} \mathrm{Tc}$-sestamibi-gated single-photon emission computed tomography (SPECT) to evaluate the final infarct size and severity at 6-month follow-up and ii) a coronary angiography at 6 months for the evaluation of IRA patency. Local ethical committee approved the study protocol, and informed written consent was obtained from the subjects. Doxycycline was provided directly from the local hospital, and the manufacturer had no role in the study.

For the present study we analyzed 73 of 110 patients of the TIPTOP trial, specifically those for which a 2D Echo-Doppler assessment including diastolic function was available also at pre-discharge (average $6 \pm 3$ days), a not pre-specified time-point of echocardiographic evaluation of the TIPTOP [7], as well as at baseline and six-month follow-up. Patients in the present sub-study had similar clinical characteristics to those included in the main trial (see Appendix A of the Supplementary Data). Both the main trial and the present sub-study were conducted in accordance with the Declaration of Helsinki.

\subsection{Echocardiographic analyses}

Two-D Echo-Doppler studies were performed with a commercially available imaging systems (Philips IE-33, Amsterdam, The Netherlands), and the following measurements were obtained according to established criteria $[1,18]$ : i) LV volumes and ejection fraction, ii) peak of early (E) and late (A) transmitral velocities and $E$-wave deceleration time (DT), iii) peak of early $\left(E^{\prime}\right)$ septal mitral annulus velocities and iiii) LV flow propagation velocity during early filling (Vp). According to the protocol of the main study [7], LV dysfunction was defined as an $\mathrm{LVEF}<40 \%$, as calculated by the on-site operator at the first echo examination in the coronary care unit immediately after primary PCI. Consistent with the current guidelines [1] on patients with LV systolic dysfunction (such as our patients), we defined a high filling pressure (HFP) pattern an $\mathrm{E} / \mathrm{A}$ ratio $\geq 2$, and a normal filling pressure (NFP) pattern an $\mathrm{E} / \mathrm{A}$ ratio $\leq 0,8$ and $\mathrm{E}<50 \mathrm{~cm} / \mathrm{s}$, respectively. For patients that fall in between, HFP was confirmed in presence of an $\mathrm{E} / \mathrm{E}^{\prime}$ ratio $>15$ [19] or an $\mathrm{E} / \mathrm{Vp}$ ratio $\geq 2,5$ [20].

\subsection{Statistical analyses}

Discrete data were summarized as frequencies, whereas continuous data were summarized as mean \pm SD or median and interquartile range, when appropriate. Differences in baseline characteristics between the two study groups were analyzed using the $\mathrm{x}^{2}$ test or Fisher's exact test for categorical variables, and the unpaired and paired, 2-tailed Student's $t$-test or Mann-Whitney test for continuous variables. Univariable and multivariable logistic regression analyses were performed to evaluate the independent contributions of pre-discharge HFP pattern. Over that baseline clinical and therapeutic variables, the changes from baseline to pre-discharge of LVEDVi, LVEF and pro-BNP release at predischarge were also tested in view of the possible interaction between the LV filling pattern and the concomitant LV remodeling process. The variables tested in the univariate mode were listed in Table 2 . For the significant variables on univariate analysis ( $p$-value $<0.05$ ) multicollinearity was assessed using collinearity diagnostics. The variance inflation factors showed no significant collinearity among these covariates $(<2.0)$. Variables with a $p$-value $<0.05$ at univariable model, were entered into the multivariable model. All tests were twosided, and a $p$-value $<0.05$ was considered statistically significant. Analyses were performed with SPSS software, version 19 (IBM Corp, Somers, NY).

\section{Results}

\subsection{Study patients}

The baseline clinical, echocardiographic, angiographic/procedural and therapeutic characteristics, of the two study groups are shown in Table 1 . There were no significant differences between the two study

Table 1

Baseline clinical and procedural characteristics of the two study groups.

\begin{tabular}{|c|c|c|}
\hline & $\begin{array}{l}\text { Doxycycline group } \\
\text { (n.37) }\end{array}$ & $\begin{array}{l}\text { Control group } \\
\text { (n. 36) }\end{array}$ \\
\hline \multicolumn{3}{|l|}{ Clinical findings } \\
\hline Age, years & 72 [62-79] & $71[60-75]$ \\
\hline Male sex, (\%) & 70 & 72 \\
\hline BAS, $\left(\mathrm{kg} / \mathrm{m}^{2}\right)$ & $1,85 \pm 0,2$ & $1,82 \pm 0,1$ \\
\hline Heart rate, (b.p.m) & $77 \pm 16$ & $79 \pm 13$ \\
\hline Systolic blood pressure, (mm Hg) & $130[110-150]$ & $130[111-150]$ \\
\hline Diabetes, (\%) & 21 & 19 \\
\hline Dyslipidaemia, (\%) & 30 & 22 \\
\hline Hypertension, (\%) & 54 & 39 \\
\hline Symptoms to door time, (min) & 145 [89-229] & $137[90-315]$ \\
\hline Pro-BNP, $(\mathrm{pg} / \mathrm{mL})$ & 575 [173-2195] & 648 [175-3177] \\
\hline Troponine I peak, $(\mu \mathrm{g} / \mathrm{ml})$ & $196 \pm 177$ & $277 \pm 192$ \\
\hline \multicolumn{3}{|l|}{ Echo-color-Doppler findings } \\
\hline E-wave peak, $(\mathrm{cm} / \mathrm{s})$ & $64 \pm 20$ & $64 \pm 19$ \\
\hline A-wave peak, $(\mathrm{cm} / \mathrm{s})$ & $81 \pm 18$ & $78 \pm 21$ \\
\hline EA ratio & $0,8 \pm 0,5$ & $0,9 \pm 0,4$ \\
\hline DT, (ms) & $194 \pm 60$ & $191 \pm 52$ \\
\hline Septal E'-wave peak, (cm/s) & $7 \pm 3$ & $7 \pm 2$ \\
\hline $\mathrm{Vp},(\mathrm{cm} / \mathrm{s})$ & $30[25-42]$ & 33 [21-48] \\
\hline High filling pressures pattern, (\%) & 30 & 17 \\
\hline $\operatorname{LVEDVi,}\left(\mathrm{mL} / \mathrm{m}^{2}\right)$ & $47[41-57]$ & $47[40-61]$ \\
\hline LVESVi, $\left(\mathrm{mL} / \mathrm{m}^{2}\right)$ & 31 [24-35] & $31[23-37]$ \\
\hline LVEF, $(\%)$ & $39[32-44]$ & $37[33-43]$ \\
\hline \multicolumn{3}{|l|}{ Angiographic and procedural findings } \\
\hline Left anterior descending artery, (\%) & 94 & 89 \\
\hline IRA TIMI flow grade $<2$ pre-PCI, (\%) & 68 & 67 \\
\hline Multivessel disease, $(\%)$ & 44 & 49 \\
\hline IRA stenting, (\%) & 100 & 100 \\
\hline Number of stents & $1,3 \pm 0,6$ & $1,4 \pm 0,6$ \\
\hline Stent length, (mm) & $20[16-31]$ & $20[16-27]$ \\
\hline IRA TIMI flow grade 3 post-PCI, (\%) & 100 & 100 \\
\hline Procedural time, (min) & $27[20-41]$ & $28[21-35]$ \\
\hline \multicolumn{3}{|l|}{ Pharmacological therapy } \\
\hline Aspirin, (\%) & 94 & 98 \\
\hline Thienopyridine, (\%) & 100 & 100 \\
\hline Abciximab, (\%) & 87 & 95 \\
\hline Statin, (\%) & 100 & 100 \\
\hline Beta-adrenergic blocker, (\%) & 94 & 83 \\
\hline ACE inhibitor/ARB antagonist, (\%) & 75 & 86 \\
\hline Mineral-receptor-antagonist, (\%) & 19 & 28 \\
\hline Loop diuretic, (\%) & 40 & 47 \\
\hline
\end{tabular}

BAS denotes body surface area, BNP, brain natriuretic peptide, E-wave, peak early ventricular filling velocity, A-wave, peak late ventricular filling velocity, DT, deceleration time of early filling velocity, $\mathrm{E}^{\prime}$ early diastolic peak velocity on the septal side of the mitral annulus, $\mathrm{Vp}$, flow propagation velocity during early filling, LVEDVI, left ventricular end diastolic volume index, LVESVi, left ventricular end systolic volume index, LVEF, left ventricular ejection fraction, IRA, infarct-related artery, TIMI, thrombolysis in myocardial infarction, $\mathrm{ACE}$, angiotensin-converting-enzyme, ARB, angiotensin II receptor blockers. Values are expressed as mean $\pm \mathrm{SD}$, or median [IQ range] or number (\%). 
groups, however it should be noted that in the doxycycline-group there was a greater incidence of hypertension, HFP pattern, left anterior descending (LAD) disease, and lower troponin I peak values.

\subsection{Left ventricular filling pattern on admission and relationship with pro- Brian natriuretic peptide}

The echo-Doppler mitral inflow velocities recorded on admission allowed to classify 19 patients as NFP pattern, and 2 patients as HFP pattern, respectively. For the remaining 52 patients with inconclusive mitral inflow velocities, the further assessment of $\mathrm{E} / \mathrm{E}^{\prime}$ or $\mathrm{E} / \mathrm{Vp}$ ratio allowed to reclassify 37 patients in the NFP group and 15 in the HFP group, respectively. The time course of plasma pro-brain natriuretic peptide (BNP) release was available in $82 \%$ of these patients. Of note, the patients in which $\mathrm{E} / \mathrm{E}^{\prime}$ and $\mathrm{E} / \mathrm{Vp}$ met the cutoff threshold for HFP showed higher values of baseline pro- BNP than their counterparts ( $2870 \mathrm{pg} / \mathrm{mL} \pm 2833$ vs. $1105 \mathrm{pg} / \mathrm{mL} \pm 1505, p=0.01$ ) despite similar values of baseline $\operatorname{LVEF}(38 \% \pm 7$ vs. $39 \% \pm 7, p=0,82)$, and troponin I peak $(263 \mu \mathrm{g} / \mathrm{L} \pm 209$ vs. $247 \mu \mathrm{g} / \mathrm{L} \pm 188, p=0.94)$, respectively.

\subsection{Early changes in LV filling pattern, volumes and ejection fraction in the two study groups}

Serial changes in LV filling pattern from baseline to pre-discharge in the two study groups are detailed in Fig. 1. From baseline to predischarge, the LV filling changed from HFP to NFP pattern in $91 \%$ of patients in the doxycycline-group, and in 67\% of those in the controlgroup, respectively. Conversely, LV filling changed from NFP to HFP pattern in $11 \%$ of patients in the doxycycline-group, and in $37 \%$ of those in the control-group, respectively. Overall, a pre-discharge HFP pattern was present in $11 \%$ of patients in the doxycycline group and in $36 \%$ of patients in the control group, respectively $(p=0.025)$.

The modifications in LV filling pattern were accompanied by a significant increase in LV volumes in the control-group compared to the doxycycline-group ( $\triangle$ LVEDVi: $10 \mathrm{ml} / \mathrm{m}^{2}$ [from -1 to $15 \mathrm{ml} / \mathrm{m}^{2}$ ] vs. $1 \mathrm{ml} / \mathrm{m}^{2}$ [from -4 to $9 \mathrm{ml} / \mathrm{m}^{2}$ ], $p=0.014 ; \Delta$ LVESVi: $4 \mathrm{ml} / \mathrm{m}^{2}$ [from
-2 to $12 \mathrm{ml} / \mathrm{m}^{2}$ ] vs. $0,4 \mathrm{ml} / \mathrm{m}^{2}$ [from -4 to $6 \mathrm{ml} / \mathrm{m}^{2}$ ], $p=0.043$ ). The LVEF increased in the doxycycline-group while decreased in the control-group. This difference was not significant, however (3\% [from -3 to $9 \%$ ] vs. $-1 \%$ [from -6 to $7 \%$ ], $p=0.183$ ).

\subsection{Independent predictors of pre-discharge high filling pressure pattern}

The univariate and multivariate regression analyses were performed to identify independent predictors of pre-discharge HFP pattern (Table 2). In addition to the baseline clinical, echocardiographic, angiographic, procedural, and therapeutic characteristics, we evaluated the peak of troponine I, as estimate of initial infarct size, and echocardiographic (LV volumes and LVEF changes from baseline to pre-discharge) and neurohormonal (pre-discharge plasma pro-BNP values) indexes which reflect a concomitant early LV remodeling process. The significant variables in the univariate analysis were entered in the multivariate model. The troponine I peak values $(p=$ $0.026)$, doxycycline therapy $(p=0.033)$, and baseline to predischarge LVEF changes ( $p=0.044$ ) were found to be independently associated with pre-discharge HFP pattern.

2.5. Relationship between pre-discharge LV filling pressure pattern and 6month echocardiographic findings

One patient in the control group with pre-discharge HFP died for heart failure in the first month after index myocardial infarction, and another patient refused to complete the echocardiographic follow-up. Thus the 6-month echocardiographic follow-up was available in 71 of 73 patients.

From baseline to 6 months, LV volumes decreased, and LVEF increased in patients with pre-discharge NFP pattern $(n=56)$ compared to their counterpart ( $n=17$ ), respectively ( $\triangle$ LVEDVi: $0.5 \mathrm{ml} / \mathrm{m}^{2}$ [from -5 to $9 \mathrm{ml} / \mathrm{m}^{2}$ ] vs. $7 \mathrm{ml} / \mathrm{m}^{2}$ [from -17 to $14 \mathrm{ml} / \mathrm{m}^{2}$ ], $p=0.08 ; \Delta$ LVESVi: $-4 \mathrm{ml} / \mathrm{m}^{2}$ [from -11 to $1 \mathrm{ml} / \mathrm{m}^{2}$ ] vs. $1.5 \mathrm{ml} / \mathrm{m}^{2}$ [from -7.5 to $12 \mathrm{ml} / \mathrm{m}^{2}$ ], $p=0.03 ; \Delta$ LVEF: $11 \%$ [from 5 to $19 \%$ ] vs. $4 \%$ [from -4 to $12 \%], p=0.04$ ), (Fig. 2). Patients with pre-discharge NFP pattern

\section{BASELINE L.V. FILLING PATTERN \\ PRE-DISCHARGE L.V. FILLING PATTERN}

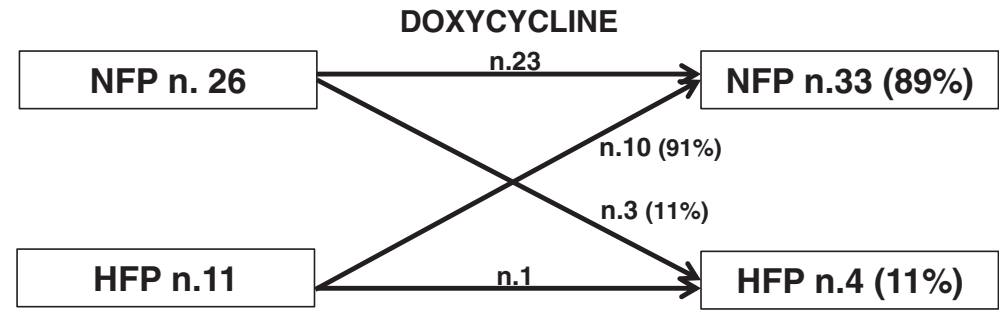

\section{CONTROL}

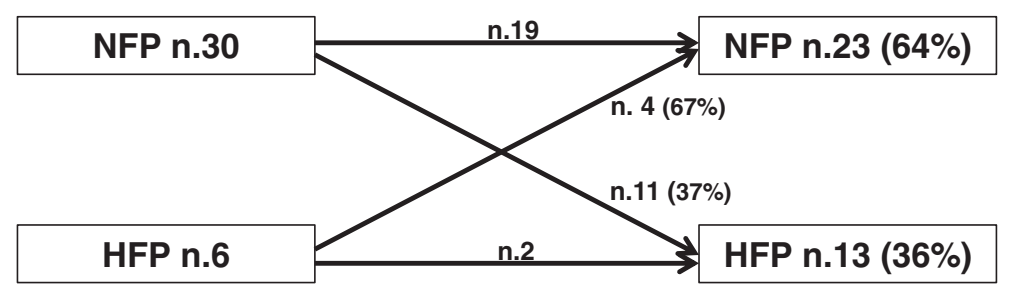

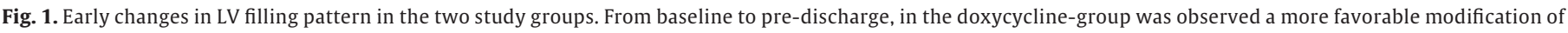

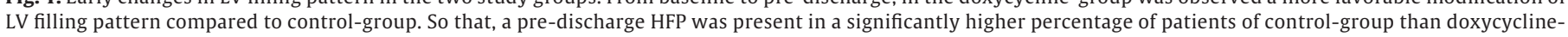
group, respectively ( $36 \%$ vs. $11 \%, p=0.025$ ). 
Table 2

Independent predictors of high filling pressure at pre-discharge.

\begin{tabular}{|c|c|c|c|c|c|c|c|c|}
\hline \multirow[b]{2}{*}{ Baseline clinical findings } & \multicolumn{4}{|c|}{ Univariate logistic regression analysis } & \multicolumn{4}{|c|}{ Multivariate logistic regression analysis } \\
\hline & $\mathrm{B}$ & $\operatorname{Exp}(B)$ & $95 \% \mathrm{CI}$ & $\mathrm{p}$ & B & $\operatorname{Exp}(B)$ & $95 \% \mathrm{CI}$ & $\mathrm{p}$ \\
\hline Age, years & 0,04 & 1,04 & 0,989-1104 & 0.114 & & & & \\
\hline Sex male, (\%) & $-1,42$ & 2,42 & $0,077-0,763$ & 0.015 & -3.966 & 0,02 & $0,000-3318$ & 0.132 \\
\hline $\mathrm{BSA},\left(\mathrm{kg} / \mathrm{m}^{2}\right)$ & -5.01 & 0,01 & $0,000-0275$ & 0.008 & -1953 & 0,142 & $0,000-4434$ & 0.712 \\
\hline Heart rate, (bpm) & 0,00 & 1,00 & $0,968-1041$ & 0.852 & & & & \\
\hline Systolic blood pressure, $(\mathrm{mm} \mathrm{Hg})$ & 0,00 & 1,00 & 0,980-1016 & 0.832 & & & & \\
\hline Diabetes, $(\%)$ & -0.23 & 1,26 & $0,363-4619$ & 0.729 & & & & \\
\hline Dyslipidaemia, (\%) & -1.17 & 0,84 & 0,237-2985 & 0.789 & & & & \\
\hline Hypertension, (\%) & -0.29 & 0,75 & $0,251-2256$ & 0.611 & & & & \\
\hline Total ischemic time, (min) & 0,002 & 1002 & 0,999-1005 & 0.193 & & & & \\
\hline \multicolumn{9}{|l|}{ Baseline echocardiographic findings } \\
\hline Baseline LVEDVi, $\left(\mathrm{mL} / \mathrm{m}^{2}\right)$ & -0.02 & 0,98 & 0,937-1029 & 0.445 & & & & \\
\hline Baseline LVESVi, $\left(\mathrm{mL} / \mathrm{m}^{2}\right)$ & -0.02 & 0,98 & 0,919-1036 & 0.423 & & & & \\
\hline Baseline LVEF, (\%) & 0,00 & 1,00 & 0,924-1091 & 0.925 & & & & \\
\hline \multicolumn{9}{|l|}{ Angiographic and procedural findings } \\
\hline IRA TIMI flow grade < 2 pre-PCI, (\%) & $-1,03$ & 0,36 & $0,092-1390$ & 0.138 & & & & \\
\hline Multivessel disease, (\%) & -0.02 & 0,98 & 0,478-1995 & 0.948 & & & & \\
\hline Stent length, (mm) & -0.03 & 0,97 & 0,919-1030 & 0.347 & & & & \\
\hline Procedural time, (min) & 0,02 & 1,03 & 0,997-1054 & 0.087 & & & & \\
\hline \multicolumn{9}{|l|}{ Pharmacological therapy } \\
\hline Abciximab, (\%) & -0.86 & 0,43 & $0,065-2779$ & 0.371 & & & & \\
\hline Beta-adrenergic blocker, (\%) & -0.25 & 0,78 & $0,182-3300$ & 0.735 & & & & \\
\hline ACE inhibitor/ARB antagonist, (\%) & -1.55 & 4,73 & $1282-17,44$ & 0.023 & 0,542 & 1,72 & $0,064-46,19$ & 0.747 \\
\hline Mineral-receptor-antagonist, (\%) & 1,17 & 3,22 & $0,986-10,515$ & 0.053 & & & & \\
\hline Loop diuretics, (\%) & 0,48 & 1,61 & $0,542-4806$ & 0.390 & & & & \\
\hline Doxycycline, (\%) & -1.54 & 0,21 & $0,062-0,742$ & 0.015 & -5113 & 0,01 & $0,000-0,666$ & 0.033 \\
\hline \multicolumn{9}{|l|}{ Enzymatic infarct size } \\
\hline Troponine I peak, $(\mu \mathrm{g} / \mathrm{L})$ & 0,01 & 1,01 & $1002-1008$ & 0.000 & 0,009 & 1,01 & $1001-1017$ & 0.026 \\
\hline \multicolumn{9}{|l|}{ Pre-discharge findings } \\
\hline Pre-discharge pro-BNP, (pg/mL) & 0,00 & 1,00 & $1000-1001$ & 0.004 & 0,001 & 1,00 & $1000-1002$ & 0.120 \\
\hline Baseline-pre-discharge LVEDVi changes, (mm) & 0,06 & 1059 & $1000-1122$ & 0.051 & -0.092 & 0,912 & $0,741-1123$ & 0.367 \\
\hline Baseline-pre-discharge LVEF changes, (mm) & -0.08 & 0,92 & $0,885-0,995$ & 0.036 & -0.255 & 0,775 & $0,604-0,994$ & 0.044 \\
\hline
\end{tabular}

Abbreviations as in Table 1 .

also showed a lower, but not significant prevalence of 6 months HFP pattern compared to patients with pre-discharge HFP pattern ( $11 \%$ vs. $25 \%, p=0.217$ ).

Remarkably, the favorable remodeling that we observed in patients with pre-discharge NFP occurred regardless of the LV filling pattern present at baseline assessment. Indeed, the 14 patients with LV filling pattern changes from HFP to NFP from baseline to pre-discharge showed a similar 6-month evolutionary changes in LV volumes in comparison to the 42 patients with NFP pattern at baseline as well as at predischarge assessment ( $\triangle$ LVEDVi: $-3 \mathrm{ml} / \mathrm{m}^{2}$ [from -12 to $8 \mathrm{ml} / \mathrm{m}^{2}$ ] vs. $1 \mathrm{ml} / \mathrm{m}^{2}$ [from -4 to $9 \mathrm{ml} / \mathrm{m}^{2}$ ], $p=0.10 ; \Delta$ LVESVi: $-7 \mathrm{ml} / \mathrm{m}^{2}$ [from -15 to $1 \mathrm{ml} / \mathrm{m}^{2}$ ] vs. $-3 \mathrm{ml} / \mathrm{m}^{2}$ [from -10 to $1 \mathrm{ml} / \mathrm{m}^{2}$ ], $p=0.24$ ).
The LVEF changes paralleled those of the LV volumes ( $\triangle$ LVEF: $13 \%$ [from 3 to $17 \%$ ] vs. $11 \%$ [from 5 to $19 \%$ ], $p=0.62$ ).

\subsection{Relationship between plasma MMPs/TIMPs, therapy and LV filling pres- sure pattern}

The baseline plasma MMPs/TIMPs were not different between the two treatment groups (see Appendix B of the Supplementary Data). However, several significant differences between the 2 study groups were found, according to the baseline echocardiographic pattern of LV filling pressures (see Appendix C of the Supplementary Data). Nominally, control-patients in HFP-pattern-group showed a significant increase in MMP-7/TIMP-3 at
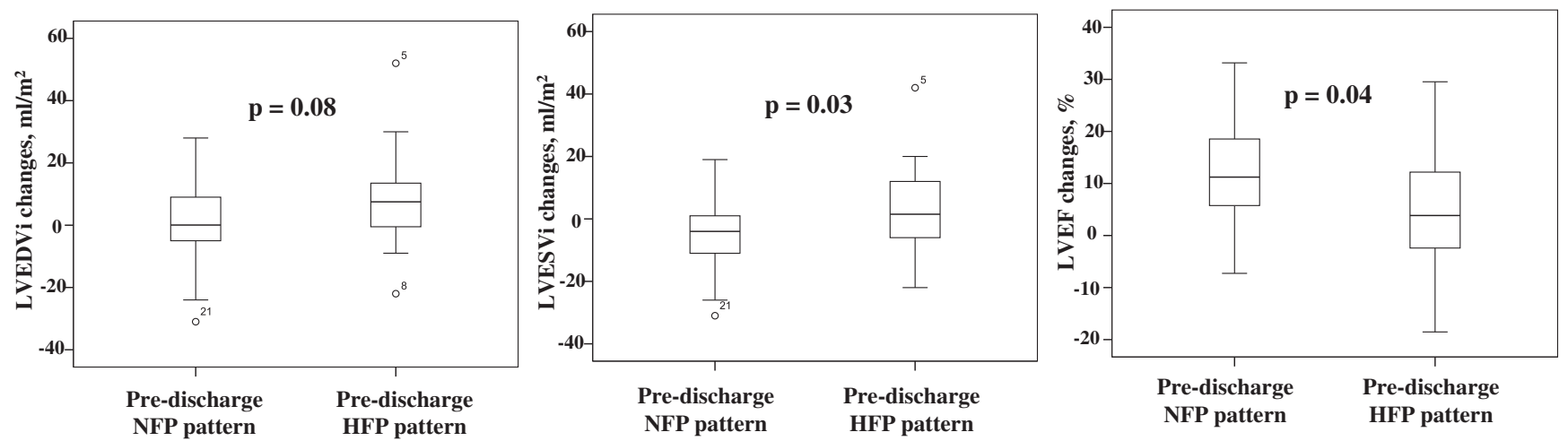

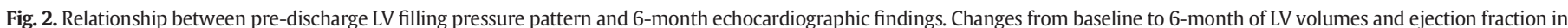

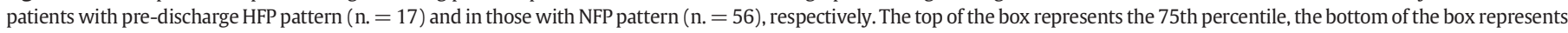

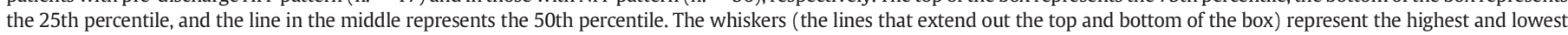
values. 
$24 \mathrm{~h}, \mathrm{MMP}-7 / \mathrm{TIMP}-1,-2,-3$ and -4 at pre-discharge and MMP-7/TIMP-1, -2 and -3 at 6 month follow-up, respectively. On the contrary, 1-month MMP9/TIMP-1 and -4 ratios, and MMP-8/TIMP-4 ratio significantly increased in doxycycline-group than control-group.

\section{Discussion}

The unique and significant findings from this study are twofold. First, a short-time treatment with doxycycline, initiated early after mechanical reperfusion in patients with STEMI and LV dysfunction, is associated with a significant improvement in LV filling pattern on echocardiographic assessment from hospital admission to pre-discharge. Importantly, this relationship was independent of the enzymatic infarct size, and concomitant LV remodeling process. Second, compared to patients with pre-discharge HFP, patients with pre-discharge NFP pattern showed less 6-month LV remodeling and, importantly, this occurred regardless of baseline LV filling pattern.

The ECM plays a major role in post-myocardial infarct remodeling [21]. Increased MMPs activation (such as MMP-1, -2, -3, -8, -9 and -14) is a well-accepted pathway leading to early ECM damage and LV remodeling [21]. The inhibition of MMPs by synthetic inhibitors has been shown to be able to reduce post-infarction adverse remodeling in animals [22-25], but not in humans [26].

For the first time, the TIPTOP study [7] has demonstrated that a timely short-term therapy with doxycycline in patients with acute STEMI and LV dysfunction significantly reduces the adverse LV remodeling for comparable definite infarct size. This effect may be due to an upregulation doxycycline-related of tissue inhibitor of MMP-2 in the first days post myocardial infarction [12]. Moreover, doxycycline possesses cytoprotective effects that are likely secondary to its capacity to act as anti-inflammatory, anti-apoptotic, reactive oxygen species scavenger, as well as MMPs inhibitor [10].

The current analysis of the TIPTOP study opens new perspectives on mechanisms through which doxycycline may determine its favorable action in STEMI patients. In fact, doxycycline therapy was associated with a favorable dynamic behavior of the LV filling during the hospital stage of acute STEMI, resulting in a significantly lower percentage of pre-discharge HFP compared to the control group.

There is no doubt that pharmacological MMP inhibition can favorably alter the course of post-myocardial infarction remodeling in term of LV volumes and function [27]. For example, in the experimental models of AMI in the rat [10] and mice [28], the treatment with broad spectrum inhibitors of MMPs (PD-166793) and doxycycline, respectively, significantly decreased LV dilation 14 days after index infarction, and yielded parallel reduction of LV end-diastolic pressure.

Because of its design, this study was unable to demonstrate any cause-effect relationships, but only correlations, which, however, allow for some interesting hypotheses. First, in control-patients with baseline HFP pattern, the early time-course of MMPs/TIMPs profile was dominated by sustained higher plasma MMP-7/TIMPs ratios than in doxycycline-patients. In adult sheep model of myocardial infarction, MMP-7 deficiency has been related to an improvement of remodeling process after localized delivery of biocomposite materials into infarcted myocardium [29]. Then again, MMP-7 has a large proteolytic portfolio [30] that can modify ECM composition and, consequently, LV architecture, and systolic and diastolic function. Our results suggest this possibility, at least in the clinical setting of STEMI and elevated LV filling pressures. Second, at 1-month follow-up doxycycline-patients with HFP pattern showed higher MMP9/TIMP-1 and -4 and MMP-8/TIMP-4 ratios than control-patients with HFP pattern. Although an early increase of MMP-9 and -8 has been related to adverse LV remodeling [9], in a later phase of myocardial infarction this same proteolytic activity can mediate wound healing [31] and preserve LV systolic function [32]. Consistently, an early short-term MMPs inhibition after experimental myocardial infarction confers a beneficial effect, while a prolonged MMPs inhibition is associated with an adverse LV remodeling [33]. Third, the MMPs/TIMPs ratios changes we observed between the two study-groups, only occurred in patients with baseline HFP pattern. Likely, in the low-risk patients with acute STEMI, such as those with NFP pattern, the powerful salvaging capacity of primary PCI with routine use of modern anti-thrombotic drugs overwhelms the benefits of additional pharmacological cardioprotective strategies, which could otherwise show benefit in patients known to be at higher risk, such as those with HFP pattern.

However, it is interesting to note that in the present study doxycycline was directly associated to a pre-discharge NFP pattern also after controlling for indexes of early concurrent LV remodeling process, namely the LVEDVi and LVEF. Thus, the question arises whether other actions different than ECM modulation can contribute to the relationship between doxycycline and diastolic function early after STEMI. Ali and Coll [15] showed both in rat and human myocardium that MMP-2 colocalizes with titin, and that cleavage of titin in rat hearts subjected to I/R injury was prevented by a selective MMP inhibitor (ONO-4817). Thus, this study highlights an interesting link between the two components responsible for myocardial stiffness: the ECM (also known to be degraded by MMPs including MMP-2) and titin filaments inside the cardiomyocyte. Metalloproteinase- 2 is responsible also for the degradation of troponin I [13] and myosin light chain [14] after I/R injury, and doxycycline has been shown to inhibit both $[13,14]$. In our study, we did not observe significant differences in MMP-2 plasma levels according to doxycycline therapy. However, since we measured the plasma MMP concentration, we cannot exclude that doxycycline may have modulated posttranslation MMP activity [34]. Anyway, the protective effect of doxycycline on titin is currently only an intriguing and plausible hypothesis.

In the present study we have observed a pre-discharge HFP pattern in $23 \%$ of patients. This finding is in agreement with previous reports in which HFP, as assessed by Doppler transmitral flow profile and/or by a short DT, ranged from 13 to $26 \%$ in non-selected patients with AMI treated with medical therapy [35-37], and occurred in $\approx 20 \%$ of 3396 patients included in the most recent MeRGE study [2]. Importantly, in the MeRGE study it was associated with a 3-fold increase in risk of death, with a considerably greater power than any previous study [2]. Our patients with predischarge NFP have a better outcome at 6-month follow-up in terms of LV remodeling compared to patients with HFP. Also this result is in agreement with previous reports [3-5], and likely provide the critical linkage between HFP and poor clinical outcome after AMI. Since the favorable outcome associated with the pre-discharge NFP pattern occurs regardless of baseline LV filling pattern, the pre-discharge period appear a good time-point for an effective assessment of diastolic function in survivors of the acute phase of a myocardial infarction.

The present analysis is subject to several limitations. The first is the lack of simultaneous hemodynamic measurement obtained with Eco-Doppler examination. The non-invasive evaluation of LV filling might be problematic to differentiate normal from pseudonormal transmitral velocity pattern. However, both E' and Vp have been shown to be reliable in detecting a pseudonormal LV filling pattern, especially in patients with LV systolic dysfunction such as our patients. An $\mathrm{E} / \mathrm{E}^{\prime}$ ratio $>15$ as well as an $\mathrm{E} / \mathrm{Vp}$ ratio $\geq 2.5$ not only predict high filling pressures with reasonable accuracy $[19,20]$, but they have also shown to be highly predictive of a poor prognosis after AMI $[38,39]$. A further support of the reliability of these indexes in classifying the proper $\mathrm{LV}$ filling pattern arises from the fact that our patients with $\mathrm{E} / \mathrm{E}^{\prime}$ ratio $>15$ or $\mathrm{E} / \mathrm{Vp} \geq 2.5$ had significantly higher values of pro-BNP respect to their counterparts [40,41], despite similar values of enzymatic infarct size and LV systolic function. Second, given the small sample size, LV filling pattern was evaluated using a binary approach, namely NFP/HFP. Therefore, HFP-group comprises patients with Echo-Doppler findings indicative of both pseudonormal and restrictive filling pattern. However, this fact might be irrelevant from a clinical point of view since both patterns have shown an unfavorable prognosis post AMI than patient without echo-Doppler findings of HFP [2]. Third, we only analyzed selected MMPs and therefore our analysis may have oversimplified the complex interplay of MMPs and TIMPs [42]. Finally, this is a post hoc analysis of a small randomized trial and the results can be considered only hypothesis generating. 
The relationship between doxycycline and post-myocardial infarct remodeling must be confirmed by double-blind study via MRI and Echo-Doppler modalities. Obviously, the impact of doxycycline on long-term clinical outcomes should be further assessed in the future with more highly powered studies.

\section{Conclusions}

In patients treated by primary PCI for a first STEMI with LV dysfunction, timely, short-term therapy with doxycycline can favorably modulate LV diastolic filling pattern during the early stage of index myocardial infarction. This may be one of the mechanisms by which doxycycline has been shown to have a favorable effect in addition to standard-of-care therapy for STEMI. To date, a major unresolved question is how to manage optimally patients with abnormal LV filling. Doxycycline can be an exciting opportunity.

Supplementary data to this article can be found online at http://dx. doi.org/10.1016/j.ijcard.2017.03.125.

\section{Funding}

The study was not supported by any public grant or industry support, none of the authors has financial disclosures.

\section{Conflict of interest}

The authors report no relationships that could be construed as a conflict of interest.

\section{References}

[1] S.F. Nagueh, O.A. Smiseth, C.P. Appleton, et al., Recommendations for the evaluation of left ventricular diastolic function by echocardiography: an update from the American Society of Echocardiography and the European Association of Cardiovascular Imaging, J. Am. Soc. Echocardiogr. 29 (2016) 277-314.

[2] J.E. Møller, G.A. Whalley, F.L. Dini, et al., Independent prognostic importance of a restrictive left ventricular filling pattern after myocardial infarction: an individual patient meta-analysis: meta-analysis research group in echocardiography acute myocardial infarction, Circulation 117 (2008) 2591-2598.

[3] P. Temporelli, P. Giannuzzi, G. Nicolosi, et al., Doppler-derived mitral deceleration time as a strong prognostic marker of left ventricular remodeling and survival after acute myocardial infarction: results of the GISSI-3 Echo substudy, J. Am. Coll. Cardiol. 43 (2004) 1646-1653.

[4] G. Cerisano, L. Bolognese, N. Carrabba, et al., Doppler-derived mitral deceleration time: an early strong predictor of left ventricular remodeling after reperfused anterior acute myocardial infarction, Circulation 99 (1999) 230-236.

[5] G. Cerisano, L. Bolognese, P. Buonamici, et al., Prognostic implications of restrictive left ventricular filling in reperfused anterior acute myocardial infarction, J. Am. Coll. Cardiol. 37 (2001) 793-799.

[6] J.E. Møller, P.A. Pellikka, G.S. Hillis, J.K. Oh, Prognostic importance of diastolic function and filling pressure in patients with acute myocardial infarction, Circulation 114 (2006) 438-444.

[7] G. Cerisano, P. Buonamici, R. Valenti, et al., Early short-term doxycycline therapy in patients with acute myocardial infarction and left ventricular dysfunction to prevent the ominous progression to adverse remodelling: the TIPTOP trial, Eur. Heart J. 35 (2014) 184-191.

[8] M.O. Griffin, G. Ceballos, F.J. Villarreal, Tetracycline compounds with non-antimicrobial organ protective properties: possible mechanisms of action, Pharmacol. Res. 63 (2011) 102-107.

[9] C.S. Webb, D.D. Bonnema, S.H. Ahmed, et al., Specific temporal profile of matrix metalloproteinase release occurs in patients after myocardial infarction: relation to left ventricular remodeling, Circulation 114 (2006) 1020-1027.

[10] F.J. Villarreal, M. Griffin, J. Omens, W. Dillmann, J. Nguyen, J. Covell, Early short-term treatment with doxycycline modulates postinfarction left ventricular remodeling Circulation 108 (2003) 1487-1492.

[11] R.A. Garcia, K.V. Go, F.J. Villarreal, Effects of timed administration of doxycycline or methylprednisolone on post-myocardial infarction remodeling inflammation and left ventricular remodeling in the rat heart, Mol. Cell. Biochem. 300 (2007) 159-169.

[12] G. Cerisano, P. Buonamici, A.M. Gori, et al., Matrix metalloproteinases and their tissue inhibitor after reperfused ST-elevation myocardial infarction treated with doxycycline. Insights from the TIPTOP trial, Int. J. Cardiol. 197 (2015) 147-153.

[13] P.Y. Cheung, G. Sawicki, M. Wozniak, W. Wang, M.W. Radomski, R. Schulz, Matrix metalloproteinase- 2 contributes to ischemia/reperfusion injury in the heart, Circulation 101 (2000) 1833-1839.

[14] G. Sawicki, H. Leon, J. Sawicka, et al., Degradation of myosin light chain in isolated rat hearts subjected to ischemia-reperfusion injury: a new intracellular target for matrix metalloproteinase-2, Circulation 112 (2005) 544-552.
[15] M.A. Ali, W.J. Cho, B. Hudson, Z. Kassiri, G. Henk, R. Schulz, Titin is a target of matrix metalloproteinase-2: implications in myocardial ischemia/reperfusion injury, Circulation 122 (2010) 2039-2047.

[16] M. Donato, V. D'Annunzio, B. Buchholz, et al., Role of matrix metalloproteinase-2 in the cardioprotective effect of ischaemic postconditioning, Exp. Physiol. 95 (2010) 274-281.

[17] A.K. Prall, G.M. Longo, W.G. Mayhan, et al., Doxycycline in patients with abdominal aneurysms and in mice: comparison of serum levels and effect on aneurysm growth in mice, J. Vasc. Surg. 35 (2002) 923-929.

[18] N.B. Schiller, P.M. Shah, M. Crawford, et al., Recommendations for quantification of the left ventricle by two dimensional echocardiography: American Society of Echocardiography Committee on standards subcommittee, J. Am. Soc. Echocardiogr. 2 (1989) 358-367.

[19] S.R. Ommen, R.A. Nishimura, C.P. Appleton, et al., Clinical utility of Doppler echocardiography and tissue Doppler imaging in the estimation of left ventricular filling pressures: a comparative simultaneous Doppler-catheterization study, Circulation 102 (2000) 1788-1794.

[20] M.J. Garcia, A.M. Ares, C. Asher, L. Rodriguez, P. Vandervoort, J.D. Thomas, An index of early left ventricular filling that combined with pulsed Doppler peak E velocity may estimate capillary wedge pressure, J. Am. Coll. Cardiol. 29 (1997) 448-454.

[21] B.I. Jugdutt, Ventricular remodeling after myocardial infarction and the extracellular collagen matrix. When is enough enough? Circulation 108 (2003) 1395-1403.

[22] F.G. Spinale, M.L. Coker, S.R. Krombach, et al., Matrix metalloproteinase inhibition during the development of congestive heart failure: effects on left ventricular dimensions and function, Circ. Res. 85 (1999) 364-376.

[23] S. Heymans, A. Luttum, D. Nuyens, et al., Inhibition of plasminogen activators or matrix metalloproteinases prevents cardiac rupture but impairs therapeutic angiogenesis and causes cardiac failure, Nat. Med. 5 (1999) 1135-1142.

[24] S. Matsumura, S. Iwanaga, S. Mochizuki, H. Okamoto, S. Ogawa, Y. Okada, Targeted selection or pharmacological inhibition of MMP-2 prevents cardiac rupture after myocardial infarction in mice, J. Clin. Invest. 115 (2005) 599-609.

[25] R. Mukherjee, C.E. Widener, T.A. Brinsa, et al., Myocardial infarct expansion and matrix metalloproteinase inhibition, Circulation 107 (2003) 618-625.

[26] M.P. Hudson, P.W. Armstrong, W. Ruzyllo, et al., Effect of selective matrix metalloproteinase inhibitor (PG-116800)to prevent ventricular remodeling after myocardial infarction. Results of thePREMIER (Prevention of Myocardial Infarction Early Remodeling) trial, J. Am. Coll. Cardiol. 48 (2006) 15-20.

[27] F.G. Spinale, Myocardial matrix remodeling and the matrix metalloproteinases: influence on cardiac form and function, Physiol. Rev. 87 (2007) 1285-1342.

[28] J.S. Ikonomidis, J.W. Hendrick, A.M. Parkhurst, et al., Accelerated LV remodeling after myocardial infarction in TIMP-1-deficient mice: effects of exogenous MMP inhibition, Am. J. Physiol. Heart Circ. Physiol. 288 (2005) H149-H158.

[29] J.A. Dixon, R.C. Gorman, R.E. Stroud, et al. Targeted regional injection of biocomposite microspheres alters post-myocardial infarction remodeling and matrix proteolytic pathways, Circulation 124 (2011) S35-S45.

[30] Y.A. Chiao, R. Zamilpa, E.F. Lopez, et al., In vivo matrix metalloproteinase-7 substrates identified in the left ventricle post-myocardial infarction using proteomics, J. Proteome Res. 9 (2010) 2649-2657.

[31] C.J. Schaffer, L.B. Nanney, Cell biology of wound healing, Int. Rev. Cytol. 169 (1996) 151-181.

[32] D. Kelly, G. Cockerill, L.L. Ng, et al., Plasma matrix metalloproteinase-9 and left ventricular remodelling after acute myocardial infarction in man; a prospective cohort study, Eur. Heart J. 28 (2007) 711-718.

[33] F.G. Spinale, G.P. Escobar, J.W. Hendrick, et al., Chronic matrix metalloproteinases inhibition following myocardial infarction in mice: differential effects of short and long-term survival, J. Pharmacol. Exp. Ther. 318 (2006) 966-973.

[34] A. Wang-Gillam, E. Siegel, D.A. Mayes, L.F. Hutchins, Y.-H. Zhou, Anti-tumor effect of doxycycline on glioblastoma cells, J. Cancer Mol. 3 (2007) 147-153.

[35] S.H. Poulsen, S.E. Jensen, K. Egstrup, Effects of long-term adrenergic beta-blockade on left ventricular diastolic filling in patients with acute myocardial infarction, Am. Heart J. 138 (1999) 710-720.

[36] J.K. Oh, Z.P. Ding, B.J. Gersh, K.R. Bailey, A.J. Tajik, Restrictive left ventricular diastolic filling identifies patients with heart failure after acute myocardial infarction, J. Am. Soc. Echocardiogr. 5 (1992) 497-503.

[37] F. Nijland, O. Kamp, A.J. Karreman, M.J. van Eenige, C.A. Visser, Prognostic implications of restrictive left ventricular filling in acute myocardial infarction: a serial Doppler echocardiographic study, J. Am. Coll. Cardiol. 30 (1997) 1618-1624.

[38] J.E. Møller, E. Søndergaard, J.B. Seward, C.P. Appleton, K. Egstrup, Ratio of left ventricular peak E-wave velocity to flow propagation velocity assessed by color Mmode Doppler echocardiography in first myocardial infarction: prognostic and clinical implications, J. Am. Coll. Cardiol. 35 (2000) 363-370.

[39] G.S. Hillis, J.E. Møller, P.A. Pellikka, et al., Noninvasive estimation of left ventricular filling pressure by $\mathrm{E} / \mathrm{e}^{\prime}$ is a powerful predictor of survival after acute myocardial infarction, J. Am. Coll. Cardiol. 43 (2004) 360-367.

[40] S. Wiese, T. Breyer, A. Dragu, et al., Gene expression of brain natriuretic peptide in isolated atrial and ventricular human myocardium: influence of angiotensin II and diastolic fiber length, Circulation 102 (2000) 3074-3079.

[41] N. Hama, H. Itoh, G. Shirakami, et al., Rapid ventricular induction of brain natriuretic peptide gene expression in experimental acute myocardial infarction, Circulation 92 (1995) 1558-1564.

[42] M.L. Lindsey, R. Zampila, Temporal and spatial expression of matrix metalloproteinases and tissue inhibitors of metalloproteinases following myocardial infarction, Cardiovasc. Ther. 30 (2012) 31-41. 